\title{
Compressive vascular syndromes
}

This series of Cardiovascular Diagnosis and Therapy examines a heterogeneous group of vascular syndromes characterized by extrinsic compression and stenosis affecting the extremities, abdominal viscera, and the cardiopulmonary system. A common thread among these disorders is that both diagnosis and therapeutic intent are driven by symptoms and secondary signs, rather than degree of stenosis or occlusion. With this in mind, we aim to provide a framework for a comprehensive clinical assessment specific to each condition.

Imaging is an essential component of diagnosis these disorders, and our review focuses heavily on the imaging basis of diagnosis as well as image-guided therapies. Recent advances in noninvasive cross-sectional imaging allow for high resolution assessment of vascular stenosis. However, real-time provocative or dynamic imaging such as arm abduction/adduction in thoracic outlet and quadrilateral space syndromes, knee flexion/extension in popliteal entrapment, inspiration/expiration in median arcuate ligament syndrome, and postural/orthostatic positioning in nutcracker syndrome are frequently helpful and often diagnostic. These can be performed with a variety of modalities, but conventional and Doppler ultrasound is especially convenient in these situations. Equivocal cases may utilize plethysmography, invasive diagnostic angiography, endovascular ultrasound, and catheter-based hemodynamic pressure measurements; again benefiting from dynamic and postural modifications during these assessments.

The thoracic outlet is prone to compression from a variety of structures. In their respective articles, Dr. Huang et al. and Dr. Habibollahi et al. examine the predisposing factors, constellation of symptoms, and therapeutic options for both arterial and venous thoracic outlet syndrome. Radiographs are useful for identifying a cervical rib or other skeletal anomaly, but Doppler ultrasound and contrast-enhanced cross-sectional imaging help confirm vascular occlusions. In both conditions, surgical decompression is mainstay of therapy with adjuvant endovascular therapy appropriate in some cases.

More peripherally, the anatomic space bordered by the teres major and minor, long head of triceps, and humeral neck known as the quadrilateral space can result in compression or injury of the axillary nerve and posterior circumflex humeral artery. Dr. Zurkiya reviews the quadrilateral space syndrome with a focus on clinical findings and imaging diagnosis. Though the syndrome is more frequently characterized by neurologic symptoms and musculoskeletal imaging findings, proximal circumflex humeral artery aneurysm formation and associated intraluminal thrombus has been associated with downstream emboli and acute ischemia.

An analogous joint space in the lower extremity is the popliteal fossa, where the popliteal artery, popliteal vein, and tibial nerve traverse a narrow window occasionally made smaller by aberrant or hypertrophied muscles or fibrous bands. Bradshaw et al. describe these variants and possible ensuing arterial injury that characterize the popliteal artery entrapment syndrome: most commonly intermittent claudication but rarely arterial thromboembolism and occlusion. Dr. Chen et al. summarize the venous popliteal entrapment syndrome, typified by limb swelling with or without venous thrombosis, and occurring in combination with the arterial syndrome in up to $15 \%$ of cases. Management depends on the etiologic subtypes of these conditions, but usually involves surgical decompression (i.e., myotomy, fascial band lysis, or tendon release) followed by surgical or transcatheter revascularization.

More proximally, the left common iliac vein is inclined to compression by the overlying right common iliac artery, predisposing to left limb swelling, venous collateralization, and iliofemoral vein thrombosis. Dr. Poyyamoli et al. in their review on May Thurner syndrome describe this common and potentially morbid condition with emphasis on acute thrombosis and prevention of post-phlebitic syndrome via rapid restoration of flow. This condition is unique from the others in that lone endovascular therapy is appropriate and effective, and often aids in diagnosis via venography and intravascular ultrasound.

The abdominal viscera are also prone to extrinsic vascular compression. Median Arcuate Ligament Syndrome can result in mesenteric arterial insufficiency and celiac artery injury. Dr. Iqbal et al. discuss the unique pathophysiology and imaging criteria of celiac compression by the median arcuate ligament and diaphragmatic crus, surgical management as a mainstay of treatment, and adjuvant endovascular therapies. Dr. Kolber et al. describe the constellation of symptoms, diagnostic criterion, and various endovascular and surgical therapies of nutcracker syndrome: left renal vein compression by the overlying superior mesenteric artery. The pathophysiology of this condition is poorly understood, with symptoms encompassing the left kidney 
(hematuria, hypertension, and orthostatic proteinuria) as well as collateral pelvic veins, sometimes associated with varicocoele, pelvic congestion syndrome, and loin pain hematuria syndrome. Debate continues on the best appropriate therapy, ranging from conservative therapy in pediatric cases, to endovascular stenting, surgical exo-vascular stenting, venous re-implantation, and left renal auto-transplantation into the iliac fossa.

Finally, Dr. Batra et al. offer a comprehensive review of a variety of cardiothoracic disorders characterized by extrinsic compression of coronary and pulmonary vessels. Several of potentially life-threatening conditions are described, including coronary compression syndrome, pulmonary vein compression, and pulmonary artery compression; any of which may arise from congenital or acquired lesions, and are characterized by a variety of imaging findings.

We hope this special series highlights the importance of multimodality imaging in diagnosis of compressive vascular syndromes, and the benefits of multidisciplinary evaluation and therapy of these conditions.

\section{Acknowledgments}

Funding: None.

\section{Footnote}

Provenance and Peer Review: This article was commissioned by the editorial office, Cardiovascular Diagnosis and Therapy for the series "Compressive Vascular Syndromes". The article did not undergo external peer review.

Conflicts of Interest: Both authors have completed the ICMJE uniform disclosure form (available at https://dx.doi. org/10.21037/cdt-21-455). The series "Compressive Vascular Syndromes" was commissioned by the editorial office without any funding or sponsorship. Drs. MKK and SK served as the unpaid Guest Editors of the series. Dr. SK serves as an unpaid editorial board member of Cardiovascular Diagnosis and Therapy from May 2019 to Apr 2021. Dr. SK reports royalties from Springer and Elsevier, consulting fees from Boston Scientific, Medtronic Inc., Penumbra Inc., Dova pharmaceuticals, US Vascular, Koo Foundation, GE healthcare, Okami Medical, grants to the institution from Angiodynamics, BD and NIH outside the submitted work; and Chair, Vascular Panel, ACR Appropriateness criteria, International Editor of Journal of Clinical Interventional Radiology. Dr. SK owns stocks in Moderna, Inovio Pharmaceuticals Inc, Ardelyx Inc, Clover health investments Corp, infinity Pharmaceuticals Inc, Novavax Inc. The authors have no other conflicts of interest to declare.

Ethical Statement: The authors are accountable for all aspects of the work in ensuring that questions related to the accuracy or integrity of any part of the work are appropriately investigated and resolved.

Open Access Statement: This is an Open Access article distributed in accordance with the Creative Commons AttributionNonCommercial-NoDerivs 4.0 International License (CC BY-NC-ND 4.0), which permits the non-commercial replication and distribution of the article with the strict proviso that no changes or edits are made and the original work is properly cited (including links to both the formal publication through the relevant DOI and the license). See: https://creativecommons.org/ licenses/by-nc-nd/4.0/. 


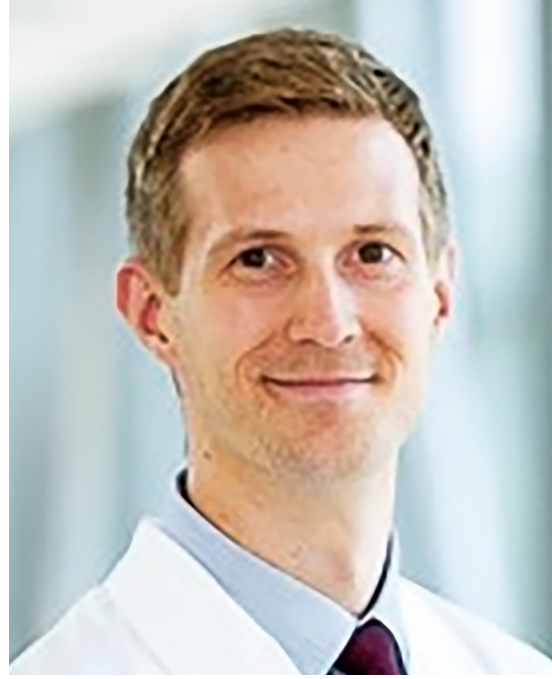

Marcin K. Kolber

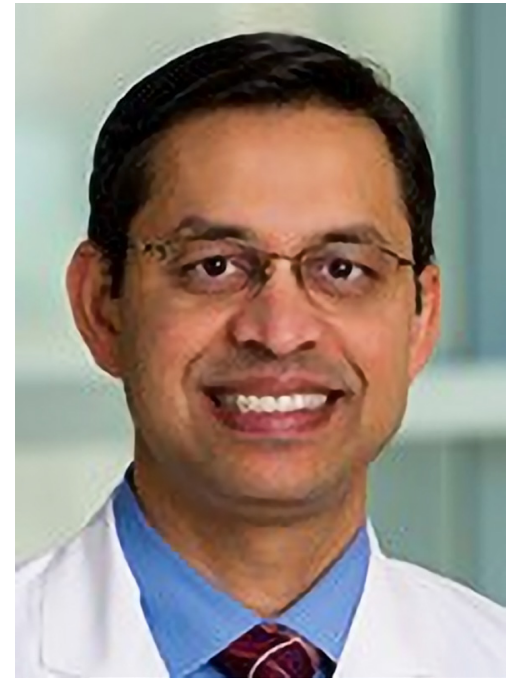

Sanjeeva Kalva

Marcin K. Kolber, MD Division of Interventional Radiology, Department of Radiology, University of Texas Soutbwestern Medical Center, Dallas, TX, USA.

(Email: marcin.kolber@utsoutbwestern.edu)

Sanjeeva Kalva, MD, FSIR, FCIRSE

Division of Interventional Radiology, Department of Radiology, Massacbusetts General Hospital, Boston, MA, USA.

(Email: skalva@mgh.harvard.edu)

Submitted Jul 22, 2021. Accepted for publication Jul 23, 2021.

doi: $10.21037 / \mathrm{cdt}-21-455$

View this article at: $10.21037 / \mathrm{cdt}-21-455$

Cite this article as: Kolber MK, Kalva S. Compressive vascular syndromes. Cardiovasc Diagn Ther 2021;11(5):1101-1103. doi: $10.21037 / \mathrm{cdt}-21-455$ 